\title{
Interaktion körperlicher und psychischer Erkrankungen
}

\author{
Fritz Hohagen \\ Klinik für Psychiatrie und Psychotherapie, Universitätsklinikum Schleswig-Holstein, Campus Lübeck, Deutschland
}

Eine Vielzahl von Studien in der Allgemeinbevölkerung und an Gruppen von Patienten mit psychischen Störungen zeigt, dass depressiv Erkrankte sowohl eine erhöhte Morbidität mit körperlichen Erkrankungen als auch eine erhöhte Mortalität aufweisen. Diese Morbidität und Mortalität bezieht sich vor allem auf zerebrovaskuläre und kardiovaskuläre Erkrankungen [Schweiger et al., 2008a]. Auch für Patienten, die einen Herzinfarkt oder Hirninfarkt erlitten haben, stellt eine sekundäre Depression einen unabhängigen Mortalitätsfaktor dar [Morris et al., 1993; Lesperance et al., 2002].

Wie erklärt sich der Zusammenhang zwischen Depression und erhöhter kardio- und zerebrovaskulärer Morbidität und Mortalität? In Frage kommen zum einen Verhaltensrisikofaktoren wie Rauchen, Alkoholabusus, verminderte Compliance und verminderte körperliche Aktivität, wie sie bei Menschen mit depressiven Erkrankungen signifikant häufiger anzufinden sind als in der Allgemeinbevölkerung. Viele dieser Risikofaktoren lassen sich durch psychoedukative und verhaltensmedizinische Interventionen verbessern. Zum anderen konnte eine ganze Reihe von physiologischen Risikofaktoren nachgewiesen werden, die das Erkrankungsund Mortalitätsrisiko bei depressiven Erkrankungen erklärt. Neben verminderter Herzratenvariabilität, erhöhter Thrombozytenaggregation und Hypercortisolismus zeigen depressiv Erkrankte ein erhöhtes Risiko für Diabetes mellitus und eine veränderte Glukoseallokation («Insulinresistenz») verglichen mit gesunden Kontrollprobanden [Weber et al., 2000; Schweiger et al., 2008b; Greggersen et al., persönliche Mitteilung]. Weitere Befunde bei depressiv Erkrankten, die die erhöhte somatische Morbidität und Mortalität erklären, sind das erhöhte viszerale Fettgewebe, das im Zusammenhang mit der Ausbildung kardio- vaskulärer Risikofaktoren gesehen wird [Everson-Rose et al., 2009] und die Manifestation eines metabolischen Syndroms, bestehend aus Hypertonus, Hyperlipidämie, diabetogener Stoffwechsellage und intraabdominellem Fettgewebe [Skilton et al., 2007]. Trotz des zwischenzeitlich erkannten Risikos für depressiv Erkrankte stellt sich gerade für Verhaltenstherapeuten eine ganze Reihe von Fragen, die wissenschaftlich weiter geklärt werden müssen. Vermindert eine erfolgreiche antidepressive Therapie das Morbiditäts- und Mortalitätsrisiko? Welche antidepressive Behandlungsmethode ist effektiv: Psychotherapie, körperliches Training oder Antidepressiva? Nachdem es sich bei den oben beschriebenen Risikokonstellationen um Prozesse handelt, die in der Regel im Verlaufe von Jahrzehnten ihre gesundheitsschädigende Wirkung entfalten, dürften Kurzinterventionen nicht ausreichen, um Morbidität und Mortalität bei depressiv Erkrankten zu senken. Dies erklärt die für Psychotherapeuten enttäuschenden Ergebnisse der ENRICHED-Studie, bei denen die verhaltenstherapeutischen Interventionen, gemessen an dem jahrzehntelangen Vorlauf der Grunderkrankung, sicherlich zu kurz gewählt waren. Neben der therapeutischen Herausforderung für die verhaltenstherapeutische Forschung und Praxis eröffnet sich für Verhaltenstherapeuten ein weites Feld in der Prävention, das ebenfalls wissenschaftlich noch unzureichend erforscht ist. Als Konsequenzen für die klinische Praxis müssen wir die Depression als Risikofaktor für körperliche Erkrankungen sehr ernst nehmen, Risikopopulationen sorgfältig ärztlich untersuchen, Depressionen frühzeitig diagnostizieren und medikamentös und psychotherapeutisch adäquat behandeln. Zusammenfassend stellt der körperliche Krankheitsaspekt bei Depressionen ein weites Forschungs- und klinisches Betätigungsfeld für die Verhaltenstherapie dar.

\section{Literatur}

Everson-Rose S, Lewis T, Karavolos K, Dugan S, Wesley D, Powell L: Depressive symptoms and increased visceral fat in middle-aged women. Psychosom Med 2009;71:410-416.

Lesperance F, Frasure-Smith N, Talajic M, Bourassa

MG: Five-year risk of cardiac mortality in relation to initial severity and one-year changes in depression symptoms after myocardial infarction. Circulation 2002;105:1049-1053.

Morris PL, Robinson RG, Andrzejewski P, Samuels J,

Price TR: Association of depression with 10-year poststroke mortality. Am J Psychiatry 1993;150:124-129.
Schweiger U, Greggersen W, Fassbinder E, Rudolf S, Steinlechner S, Nagel M, Sipos V: Interaktion von Depression und körperlichen Erkrankungen. Psychiatrie Psychotherapie up2date 2008a;2:21-31.

Schweiger U, Greggersen W, Rudolf S, Pusch M,

Menzel T, Winn S, Hassfurth J, Fassbinder E, Kahl KG, Oltmanns K, Hohagen F, Peters A: Disturbed glucose disposal in patients with major depression; application of the glucose clamp technique. Psychosom Med 2008b;70:170-176.
Skilton MR, Moulin P, Terra JL, Bonnet F: Associations between anxiety, depression, and the metabolic syndrome. Biol Psychiatry 2007;6:1251-1257.

Weber B, Schweiger U, Deuschle M, Heuser I: Major

depression and impaired glucose tolerance. Exp Clin Endocrinol Diabetes 2000;108:187-190.

\section{KARGER}

Fax +497614520714

Information@Karger.de

www.karger.com (c) 2010 S. Karger GmbH, Freiburg

Accessible online at:

www.karger.com/ver
Prof. Dr. Fritz Hohagen

Klinik für Psychiatrie und Psychotherapie

Universitätsklinikum Schleswig-Holstein, Campus Lübeck

Ratzeburger Allee 160, 23538 Lübeck

Tel. +49 451 500-2440, Fax -5097

fritzhohagen@psychiatrie.uk-sh.de 